\title{
Lettre du président
}

\section{G. Rougereau}

(C) Springer-Verlag France 2010

Chers amis,

Les temps changent, et la Société de médecine et chirurgie du pied s'adapte et s'organise. Bien sûr nous sommes toujours fidèles à cette idée de grand Pied, cette formidable et magnifique organisation qui permet d'avancer. Nous aussi nous avançons. Dans cette vie de plus en plus dense, rapide et éclatée, regrouper l'ensemble des acteurs qui traitent l'ensemble des pathologies du pied et de la cheville nous semble actuel et indispensable. C'est pourquoi nous restons fédérateurs autour de cet organe, équerre de support de notre être.

Associés aux médecins, rhumatologues ou chirurgiens qui font la base de notre groupe, au cours de notre dernier congrès, nous avions sollicité de nombreux spécialistes en dehors de notre société qui nous ont apporté leur expérience sur des pathologies appliquées aux pieds et aux chevilles. Et nous continuerons d'ouvrir notre débat à d'autres thérapeutes qui partageront leurs travaux avec nous comme les angiologues, dermatologues, neurologues, médecins de médecine physique, radiologues, pédiatres, endocrinologues, diabétologues, etc.

Nous avons eu aussi beaucoup de plaisir et d'intérêt à accueillir les podologues, qu'ils soient étudiants ou chevronnés, à ce dernier congrès du mois de décembre, comme les autres années.

La preuve de l'intérêt constant d'une vision large des pathologies du pied et de la cheville est que nous sommes de plus en plus nombreux au sein de la Société française de médecine et chirurgie du pied (SFMCP), et cela juste après avoir fêté ses 40 ans. J'ai l'immense honneur et plaisir de succéder aux past-présidents qui ont su faire vivre et prospérer notre société, Bernard Daum, Christine Thémar-Noël, Patrice Diebold... La SFMCP ne serait pas aussi florissante sans les efforts sans cesse renouvelés de son trésorier Didier Mainard et de tous les membres du bureau. Je souhaite transmettre le plus beau flambeau possible, grâce à vous tous, au premier vice-président Maurice Bouysset, respectant la tradition du médecin qui remplace le chirurgien et vice versa.

Les congrès et les réunions de toutes sortes se multiplient. C'est pourquoi nous avons décidé, en réunion de bureau, de concentrer nos efforts sur un congrès annuel, qui se tiendra à Paris, le deuxième week-end de décembre, comme à son habitude. Ce congrès débutera le vendredi à midi et se poursuivra jusqu'au samedi soir. La société suivra comme à son habitude d'autres réunions et d'autres congrès, aussi bien en France qu'à l'étranger, où elle participera activement en organisant tables rondes et communications, témoins des travaux de ses membres et de la société.

Nous renouvelons ici notre attachement, ancien et de longue date, à une collaboration avec d'autres sociétés comme l'EFAS (European Foot and Ankle Society) et l'IFFAS (International Federation of Foot and Ankle Societies).

Nous communiquerons ensemble de plus en plus, via le site Internet piloté par Philippe Jouet et la revue de la société supportée par Paul-Hubert Benamou. N'hésitez pas à nous rejoindre sur le site qui est de plus en plus notre lien avec les membres de la SFMCP, mais aussi avec tous ceux que le pied et la cheville intéressent.

En vous souhaitant à tous une très belle année 2010, de travail et de joies, sous le signe de la réunion des compétences.

G. Rougereau $(\bowtie)$

Rue de Rémusat, F-75116 Paris, France

e-mail : guillaume.rougereau@wanadoo.fr 